\title{
El efecto de la autoeficacia y el trabajo colaborativo en estudiantes novatos de programación
}

\author{
The effect of self-efficacy and peer collaboration in novice \\ programming students
}

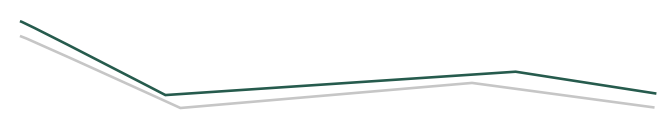

Carlos Argelio Arévalo Mercado*凶, Estela Lizbeth Muñoz Andrade**, Juan Manuel Gómez Reynoso**

Arévalo Mercado, C. A., Muñoz Andrade, E. L., \& Gómez Reynoso, J. M. (2018). El efecto de la autoeficacia y el trabajo colaborativo en estudiantes novatos de programación. Investigación y Ciencia de la Universidad Autónoma de Aguascalientes, 26(74), 73-80.

RESUMEN

El aprendizaje de la programación es complicado para alumnos que inician una carrera relacionada con las tecnologías de información. Se han identificado factores significativos como la experiencia previa, los modelos mentales incorrectos y la habilidad para las matemáticas, entre otros. También se ha identificado una relación estrecha entre el desempeño académico y los procesos cognitivos, metacognitivos y motivacionales. En este estudio se intentó identificar las variables motivacionales y metacognitivas que influyen en los estudiantes universitarios que inician el aprendizaje de la programación. Se utilizó el instrumento MSLQ para recolectar información de una muestra aleatoria de 110 alumnos cursando programación introductoria en la UAA. Se encontró que aunque los alumnos percibieron como importante el valor de la tarea, la creencia de control y la orientación

Palabras clave: programación; motivación; metacognición; autoeficacia; aprendizaje colaborativo. Keywords: programming; motivation; metacognition; self-efficacy; collaborative learning.

Recibido: 7 de septiembre de 2017, aceptado: 28 de marzo de 2018

* Departamento de Sistemas de Información, Centro de Ciencias Básicas, Universidad Autónoma de Aguascalientes. Av. Universidad 940, Ciudad Universitaria, C. P. 20131, Aguascalientes, Ags., México. Correo electrónico: carlos.arevalo@edu.vaa.mx

* Departamento de Sistemas Electrónicos, Centro de Ciencias Básicas, Universidad Autónoma de Aguascalientes. Av. Universidad 940, Ciudad Universitaria, C. P. 20131, Aguascalientes, Ags., México. Correo electrónico: elmunoz@correo.vaa.mx; jmgr@correo.vaa.mx

$凶$ Autor para correspondencia intrínseca a la tarea, el modelo de regresión lineal aplicado indicó que las variables que predicen en mayor medida el rendimiento académico fueron la autoeficacia y el trabajo colaborativo.

ABSTRACT

Learning to program is a difficult task for students starting a bachelor's degree related to information technology. Factors such as previous experience, mental models and mathematical skills have been identified as relevant. It has also been identified that motivation, cognitive and metacognitive processes have a close relation to academic performance. This study tried to identify significant motivational and metacognitive variables associated to academic performance in learning to program. We used the MSLQ questionnaire to collect information of a random sample of 110 first semester students of introductory programming in UAA. It was found that even though students identified variables such as task value, control beliefs and intrinsic goal orientation as important to them, the applied regression model indicated that self-efficacy and peer collaboration were the significant predictors of performance.

\section{INTRODUCCIÓN}

El aprendizaje de la programación es una habilidad básica que los estudiantes universitarios deben adquirir durante los primeros semestres de carreras relacionadas con las ciencias computacionales. La programación se relaciona directamente con la 
IIVESTIGACIÓn Y CIERCIA DE LA UNIVERSIDAD AUTÓNOMA

capacidad pensar de manera abstracta y de resolver problemas creando algoritmos que se convertirán posteriormente en aplicaciones de software. Sin embargo, evidencia tanto empírica (Blackwell, 2002; Jenkins, 2002; Mow, 2008) como anecdótica (Dehnadi \& Bornat, 2006; Milne \& Rowe, 2002) indica que los estudiantes que inician enfrentan serias dificultades para adquirir tales habilidades.

El problema del aprendizaje de la programación ha sido extensamente estudiado desde diversas perspectivas en los últimos 20 años, tanto para entender la propia naturaleza del problema (Baldwin \& Macredie, 1999; Bornat, Dehnadi, \& Simon, 2008), como para proponer alternativas tecnológicas (Arévalo Mercado, Muñoz Andrade, \& Gómez Reynoso, 2011 ; Cooper, Dann, \& Pausch, 2000; Maries \& Kumar, 2007) e instruccionales (Skubch \& Thielscher, 2005) que busquen mejorar el proceso de enseñanza-aprendizaje de la programación.

En el ámbito de la psicología cognitiva se han estudiado variables relacionadas con los atributos de los estudiantes de programación, tales como la autoeficacia, la habilidad para las matemáticas y los modelos mentales, entre otras (Byrne \& Lyons, 2001). El objetivo de estas investigaciones ha sido tratar de identificar predictores de desempeño, que a su vez permitan un diseño instruccional enfocado a las características individuales de los estudiantes. También existe una corriente importante de investigaciones asociadas al concepto de la metacognición, que se entiende como la conciencia de los propios procesos cognitivos (Alexander, 2008; Flavell, 1979). La metacognición se relaciona directamente con la capacidad de solución de problemas en tanto ésta permite identificar objetivos, seleccionar o corregir estrategias de solución y monitorear el avance hacia la solución.

Por otro lado, se ha encontrado que los procesos cognitivos y metacognitivos están a su vez vinculados a la variable conductual de la motivación (Mayer, 1988, 1998, 2010) . La motivación como concepto ha sido estudiada con detalle en las ciencias de la conducta y en la educación. Al nivel más básico, se habla de una dicotomía en donde la motivación puede ser intrínseca (basada en realizar algo que al sujeto le es inherentemente interesante y disfrutable) o extrínseca (basada en estímulos y situaciones externas). Autores clásicos (Dewey, 1913,1933 ) y modernos (Ryan \& Deci, 2000; Schiefe- le, 1991) resaltan la diferencia cualitativa que se da en el aprendizaje cuando éste se basa en el interés personal o en la coerción externa.

En el contexto cotidiano de las aulas los profesores de programación se enfocan a enseñar a los estudiantes modelos mentales y estrategias de solución de problemas (es decir, procesos cognitivos) mediante el modelo clásico teoría-ejemplo-ejercicio y se asume que al utilizar este modelo se obtendrán como subproducto las habilidades metacognitivas. Sin embargo, se ha encontrado que tales habilidades deben enseñarse explícitamente (Volet \& Lund, 1994).

En este estudio se midió el grado de motivación de los estudiantes que inician cursos de programación en la Universidad Autónoma de Aguascalientes (UAA) y se buscaron correlaciones entre la motivación, las habilidades metacognitivas y el desempeño académico.

El objetivo general del estudio consistió en Identificar si existe una correlación entre la motivación intrínseca, la habilidad metacognitiva y el desempeño académico de los estudiantes novatos de programación de la UAA; es decir, identificar si los alumnos intrínsecamente motivados para llevar a cabo una tarea exhiben a su vez habilidades metacognitivas o si estos factores son independientes entre sí como predictores del desempeño académico en programación o, más aun, si otros son los factores que predicen mejor este desempeño.

\section{MATERIALES Y MÉTODOS}

Se utilizaron 15 variables, obtenidas de 81 preguntas del instrumento MSLQ (Motivated Strategies for Learning Questionnaire) (Pintrich, Smith, Garcia, \& McKeachie, 1991) seleccionado por su consistencia interna (Barak, Kastelan, \& Azia, 2016; Dent \& Koenka, 2016; Wong, Chai, Chen, \& Chin, 2013) y aplicabilidad al tema de la motivación y la metacognición en el aprendizaje. La descripción operativa de estas variables (tabla 1 y tabla 2) puede encontrarse en Kuhl (2000), así como en Pintrich et al. (1991). En las conclusiones de este reporte se describen las variables que tuvieron efecto significativo en el rendimiento académico. La escala de calificación fue de tipo Likert, de un rango de 1 a 7 , en donde el valor 1 se define como "No tiene en absoluto nada que ver conmigo", el valor 2 es "Me describe muy poco", el 3 corresponde a "Me describe moderadamente", el valor 4 es "No estoy seguro 
Tabla 1

Constructos relacionados con la motivación del aprendizaje

\begin{tabular}{clll}
\hline No. & \multicolumn{1}{c}{ Subescala } & \multicolumn{1}{c}{ Abrev. } & \multicolumn{1}{c}{ Ítems } \\
\hline 1 & Orientación a metas intrínsecas & Intr & $1,16,22,24$ \\
2 & Orientación a metas extrínsecas & Extr & $7,11,13,30$ \\
3 & Valor de la tarea & Tskv & $4,10,17,23,26,27$ \\
4 & Creencia de control & Cont & $2,9,18,25$ \\
5 & Autoeficacia para el & Self & $5,6,12,15,20,21,29,31$ \\
& aprendizaje & Tanx & $3,8,14,19,28$ \\
6 & Ansiedad de examen & Tal
\end{tabular}

Nota: Con datos de Pintrich et al. (1991).

Tabla 2

Constructos relacionados con estrategias de aprendizaje

\begin{tabular}{clll}
\hline No. & \multicolumn{1}{c}{ Subescala } & \multicolumn{1}{c}{ Abrev. } & \multicolumn{1}{c}{ Ítems } \\
\hline 7 & Ensayo & Reh & $39,46,59,72$ \\
8 & Elaboración & Elab & $53,62,64,67,69,81$ \\
9 & Organización & Org & $32,42,49,63$ \\
10 & Pensamiento crítico & Crit & $38,47,51,66,71$ \\
11 & Autorregulación metacognitiva & Meta & $33,36,41,44,55,56,57,61,76,78,79$ \\
12 & Tiempo y medio ambiente de & Tsdy & $35,43,52,65,70,73,77,80$ \\
& estudio & & $37,48,60,74$ \\
13 & Regulación del esfuerzo & Eff & $34,45,50$ \\
14 & Aprendizaje por pares & Peer & $40,58,68,75$ \\
15 & Búsqueda de ayuda & Help &
\end{tabular}

Nota: Con datos de Pintrich et al. (1991).

(a)", el 5 es "Me describe suficientemente", el 6 "Me describe mucho" y el 7 "Me describe totalmente".

También se incluyó la variable calificación (CAL) como variable dependiente, cuya definición operacional es el rendimiento académico de los alumnos participantes durante la materia de Algoritmos y Programación. La medición de ésta fue mediante exámenes estandarizados por la academia de programación del Departamento de Sistemas Electrónicos. En la sección de resultados puede observarse que el comportamiento de esta variable fue normal (figura 1). El estilo de enseñanza fue controlado mediante la impartición de la materia por un mismo instructor en los grupos participantes.

En los datos obtenidos en la prueba piloto (aplicada en noviembre 2015 y en mayo 2016) se detectó la necesidad de realizar ajustes al instrumento MSLQ en las subescalas de Regulación del esfuerzo $(\alpha=0.58$, estudio noviembre 2015, $\alpha=0.21$, estudio mayo 2016) y la de Búsqueda de ayuda ( $\alpha=0.15$, estudio noviembre 2015, $\alpha=0.42$, estudio mayo 2016), ambas con valores inferiores a la confiabilidad indicada por la literatura (superior a 0.6). De las 15 subescalas que conforman el instrumento, las dos antes mencionadas se revisaron y su redacción fue ajustada buscando mejorar su claridad y consistencia.

Durante noviembre de 2016 se seleccionaron 140 alumnos de manera aleatoria de las carreras de Ingeniero en Sistemas Computacionales (ISC) e Ingeniero en Electrónica (IE) que cursaban la materia de Algoritmos y Programación. De los 140 alumnos seleccionados, 110 completaron la encuesta. La misma se aplicó después de su segundo examen parcial y antes del examen final, con el objetivo de que ellos fuesen capaces de identificar sus estrategias de aprendizaje durante los primeros meses del curso y el nivel de motivación hacia el final del mismo. Los valores Alfa de Cronbach resultantes 


\section{DE AGUASCALIERTES}

Tabla 3

Valores Alfa de Cronbach de escala de motivación

\begin{tabular}{lcr}
\hline \multicolumn{1}{c}{ Subescala } & Clave & Alpha de Cronbach \\
\hline Orientación intrínseca & Intr & 0.76 \\
Orientación extrínseca & Extr & 0.70 \\
Valor de la tarea & Tskv & 0.85 \\
Creencia de control & Cont & 0.64 \\
Autoeficacia & Slfef & 0.88 \\
Ansiedad a examen & Tanx & 0.84 \\
\hline
\end{tabular}

Nota: Elaboración propia.

Tabla 4

Valores Alfa de Cronbach de escala de aprendizaje

\begin{tabular}{lcr}
\hline \multicolumn{1}{c}{ Subescala } & Clave & Alpha de Cronbach \\
\hline Ensayo & Rhr & 0.63 \\
Elaboración & Elab & 0.62 \\
Organización & Org & 0.70 \\
Pensamiento crítico & Thk & 0.62 \\
Autorregulación metacognitiva & Meta & 0.82 \\
Tiempo y ambiente de estudio & Env & 0.76 \\
Regulación del esfuerzo & Eff & 0.69 \\
Aprendizaje colaborativo & Peer & 0.67 \\
Búsqueda de ayuda & Hlp & 0.71 \\
\hline Nota: Elaboración propia & &
\end{tabular}

de la aplicación de la encuesta se muestran en la tabla 3 y en la tabla 4 . En todos los casos, los valores se consideran en el rango de confiabilidad de aceptables.

\section{RESULTADOS}

La estadística descriptiva resultante de la aplicación de la encuesta $(n=110)$ se muestra en la tabla 5 . La media de la variable calificación fue de 6.28, la cual de acuerdo a la escala de la UAA no es aprobatoria. Las subescalas con mayor valor obtenido fueron TskV (5.89, valor percibido de la tarea), seguida de Cont (5,42, Creencia de control) e Intr (5.33, Orientación intrínseca al objetivo). Las subescalas del grupo de estrategias de aprendizaje (tabla 2 ) recibieron un valor menor en su conjunto, comparadas con las de la escala de motivación (tabla 1). De éstas últimas, la variable Eff (regulación del esfuerzo) fue en promedio la de mayor calificación (4.99). Puede verse que las respuestas caen en el rango de 4 y 5 ; es decir, las categorías de "No estoy seguro" y "Me describe suficientemente".

Los contenidos académicos del instrumento de medición aplicado (segundo examen parcial) fueron diseñados y estandarizados por la Academia de programación del Departamento de Sistemas Electrónicos. Los temas incluyeron: estructuras de control, manejo de arreglos y construcción de funciones, de los cuales se solicitó a los alumnos la elaboración de programas sencillos. De la muestra recolectada, $44 \%$ de los participantes obtuvieron una calificación reprobatoria (48/110), lo cual es consistente con el histórico de porcentajes de reprobación de la UAA y con la literatura (Mahmoud, Dobosiewicz, \& Swayne, 2004) . Resulta llamativo el alto porcentaje de alumnos que obtuvieron una calificación igual o mayor a $9(34 \%, 37 / 110)$. 


\section{IIVESTIGAGIÓn Y CUERCIA DE LA UחIVERSIDAD AUTÓNOMA

Tabla 5

Estadística descriptiva. Subescalas de motivación y de aprendizaje

Estadísticos descriptivos

\begin{tabular}{|l|c|r|r|}
\hline & Media & $\begin{array}{c}\text { Desviación } \\
\text { típica }\end{array}$ & \multicolumn{1}{c|}{$\mathrm{N}$} \\
\hline CAL & 6.2800 & 3.32853 & 110 \\
INTR & 5.3386 & 1.00320 & 110 \\
EXTR & 5.1818 & 1.13781 & 110 \\
TSKV & 5.8924 & .93510 & 110 \\
CONT & 5.4295 & .95130 & 110 \\
SELF & 5.3159 & .94209 & 110 \\
TANX & 4.3327 & 1.47301 & 110 \\
REH & 4.4000 & 1.19690 & 110 \\
ELAB & 4.4394 & .99636 & 110 \\
ORG & 4.1455 & 1.28879 & 110 \\
CRIT & 4.6018 & .99337 & 110 \\
META & 4.8205 & .92612 & 110 \\
TSDY & 4.6762 & .72495 & 110 \\
EFF & 4.9909 & .99536 & 110 \\
PEER & 4.2333 & 1.37218 & 110 \\
HLP & 4.3977 & 1.02620 & 110 \\
\hline
\end{tabular}

Nota: Elaboración propia.

En lo que respecta a las subescalas de estrategias de aprendizaje (tabla 5), los alumnos otorgaron también en ambas categorías una mayor valoración en promedio a la variable Eff (regulación del esfuerzo), la cual tiene que ver con la autorregulación del esfuerzo del alumno con respecto a distractores externos y el enfoque que se da a tareas poco interesantes o laboriosas. Ésta

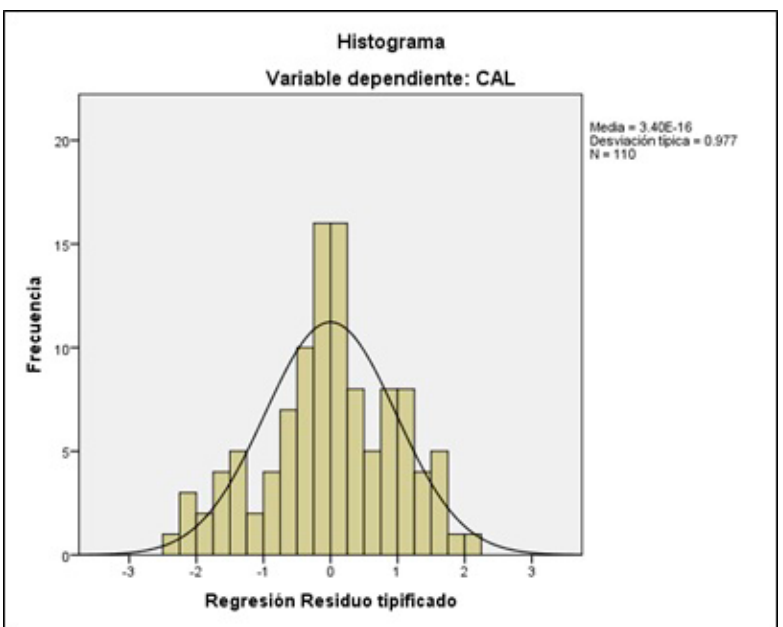

Figura 1. Comportamiento de la variable Calificación (CAL).

Elaboración propia.

refleja el compromiso hacia los objetivos, a pesar de la dificultad de la tarea.

En la escala de aprendizaje del MSLQ, los alumnos consideraron como una de las menos importantes a la variable Peer (aprendizaje por pares). Esto es llamativo, ya que en el modelo de regresión obtenido (tabla 6) para el conjunto de casos analizados, la variable Peer forma parte de las variables predictoras y su valor Beta $(\beta=0.283)$ es la que se interpreta como mejor predictor (aunque por un pequeño margen) del desempeño académico (variable CAL).

El modelo obtenido mediante análisis de regresión lineal (tabla 6) dio como resultado cinco

Tabla 6

Modelo de variables predictoras de variable dependiente CAL

Resumen del modelo ${ }^{t}$

\begin{tabular}{|c|c|c|c|c|c|c|c|c|c|c|}
\hline \multirow[b]{2}{*}{ Modelo } & \multirow[b]{2}{*}{$\mathrm{R}$} & \multirow[b]{2}{*}{ R cuadrado } & \multirow[b]{2}{*}{$\begin{array}{c}\text { R cuadrado } \\
\text { corregida }\end{array}$} & \multirow[b]{2}{*}{$\begin{array}{c}\text { Error típ. de la } \\
\text { estimación }\end{array}$} & \multicolumn{5}{|c|}{ Estadísticos de cambio } & \multirow[b]{2}{*}{$\begin{array}{l}\text { Durbin- } \\
\text { Watson }\end{array}$} \\
\hline & & & & & $\begin{array}{l}\text { Cambio en R } \\
\text { cuadrado }\end{array}$ & Cambio en F & gl1 & $\mathrm{g} \mid 2$ & $\begin{array}{l}\text { Sig. Cambio } \\
\text { en F }\end{array}$ & \\
\hline 1 & $.308^{2}$ & .095 & .086 & 3.18147 & .095 & 11.309 & 1 & 108 & .001 & \\
\hline 2 & $.405^{b}$ & .164 & .148 & 3.07190 & .069 & 8.842 & 1 & 107 & .004 & \\
\hline 3 & $.481^{\mathrm{c}}$ & .231 & .210 & 2.95931 & .067 & 9.297 & 1 & 106 & .003 & \\
\hline 4 & $.547^{d}$ & .299 & .273 & 2.83862 & .068 & 10.205 & 1 & 105 & .002 & \\
\hline 5 & $.575^{e}$ & .330 & .298 & 2.78905 & .031 & 4.765 & 1 & 104 & .031 & 1.962 \\
\hline
\end{tabular}

a. Variables predictoras: (Constante), TANX

b. Variables predictoras: (Constante), TANX, PEER

c. Variables predictoras: (Constante), TANX, PEER, EXTR

d. Variables predictoras: (Constante), TANX, PEER, EXTR, SELF

e. Variables predictoras: (Constante), TANX, PEER, EXTR, SELF, EFF

f. Variable dependiente: CAL

Nota: Elaboración propia. 
Tabla 7

Detalle de coeficientes del modelo de regresión lineal

\begin{tabular}{|c|c|c|c|c|c|c|c|c|c|c|c|c|c|}
\hline \multicolumn{14}{|c|}{ Coeficientes ${ }^{\mathrm{a}}$} \\
\hline \multirow{2}{*}{\multicolumn{2}{|c|}{ Modelo }} & \multicolumn{2}{|c|}{ Coeficientes no estandarizados } & \multirow{2}{*}{$\begin{array}{c}\begin{array}{c}\text { Coeficientes } \\
\text { tipificados }\end{array} \\
\text { Beta }\end{array}$} & \multirow[b]{2}{*}{$t$} & \multirow[b]{2}{*}{ Sig. } & \multicolumn{2}{|c|}{$\begin{array}{c}\text { Intervalo de confianza de } 95.0 \% \\
\text { para B }\end{array}$} & \multicolumn{3}{|c|}{ Correlaciones } & \multicolumn{2}{|c|}{ Estadisticos de colinealidad } \\
\hline & & B & Error tip. & & & & Limite inferior & $\begin{array}{l}\text { Limite } \\
\text { superior }\end{array}$ & Orden cero & Parcial & Semiparcial & Tolerancia & $\mathrm{FN}$ \\
\hline \multirow[t]{2}{*}{1} & (Constante) & 9.294 & .946 & & 9.822 & .000 & 7.419 & 11.170 & & & & & \\
\hline & TANX & .696 & .207 & -.308 & -3.363 & .001 & -1.106 & .286 & .308 & -.308 &,- 308 & 1.000 & 1.000 \\
\hline \multirow[t]{3}{*}{2} & (Constante) & 7.033 & 1.189 & & 5.915 & .000 & 4.676 & 9.389 & & & & & \\
\hline & TANX & .808 & .203 & .357 & -3.973 & .000 & -1.211 & .405 & .308 & $\cdot .359$ &. .351 & .966 & 1.036 \\
\hline & PEER & .649 & .218 & .267 & 2.973 & .004 & .216 & 1.081 & .201 & .276 & .263 & 966 & 1.036 \\
\hline \multirow[t]{4}{*}{3} & (Constante) & 9.683 & 1.438 & & 6.735 & .000 & 6.832 & 12.533 & & & & & \\
\hline & TANX & .579 & .210 & .256 & -2.759 & .007 & .994 &. .163 & .308 & $\cdot 259$ & .235 & .842 & 1.188 \\
\hline & PEER & .847 & .220 & .349 & 3.851 & .000 & .411 & 1.284 & .201 & .350 & .328 & .881 & 1.135 \\
\hline & EXTR & .865 & .284 &. .296 & -3.049 & .003 & -1.428 & .303 & .278 & -.284 &. .260 & .771 & 1.297 \\
\hline \multirow[t]{5}{*}{4} & (Constante) & 4.942 & 2.026 & & 2.439 & .016 & .925 & 8.959 & & & & & \\
\hline & TANX & -.257 & .225 & -.114 & -1.143 & .255 & -703 & .189 & -.308 & -111 & -093 & .673 & 1.485 \\
\hline & PEER & .713 & .215 & .294 & 3.312 & .001 & .286 & 1.140 & .201 & .308 & .271 & .847 & 1.180 \\
\hline & EXTR & -1.222 & .294 & -418 & -4.154 & .000 & -1.806 & .639 & .278 & -.376 & -.339 & .660 & 1.516 \\
\hline & SELF & 1.085 & 340 & .307 & 3.195 & .002 & .412 & 1.759 & .290 & .298 & .261 & .722 & 1.385 \\
\hline \multirow[t]{6}{*}{5} & (Constante) & 2.573 & 2.267 & & 1.135 & .259 & -1.922 & 7.069 & & & & & \\
\hline & TANX & $\cdot .165$ & .225 & .073 & -.735 & .464 & .612 & .281 & -308 & .072 & .059 & .650 & 1.539 \\
\hline & PEER & .686 & .212 & .283 & 3.237 & .002 & .266 & 1.106 & .201 & .303 & .260 & .844 & 1.184 \\
\hline & EXTR & -1.282 & .290 & .438 & -4.414 & .000 & -1.857 & .706 & .278 & $\cdot 397$ & .354 & .654 & 1.529 \\
\hline & SELF & .945 & .340 & .267 & 2.781 & .006 & .271 & 1.619 & .290 & .263 & .223 & .696 & 1.436 \\
\hline & EFF & .628 & .288 & .188 & 2.183 & .031 & .058 & 1.199 & .273 & .209 & .175 & .869 & 1.151 \\
\hline
\end{tabular}

a. Variable dependiente: CAL

Nota: Elaboración propia.

variables que predicen el comportamiento de la variable dependiente CAL: Tanx, Peer, Extr, Self y Eff. Las 10 variables restantes no contribuyen al comportamiento de la variable dependiente.

En la tabla 7 se muestran los coeficientes del modelo de regresión lineal, en donde se observa que de los valores Beta de las variables predictoras (renglón 5), la variable predictora con mayor aportación al modelo es Peer $(\beta=0.283)$, seguida de Self $(\beta=0.267)$. Las variables Tanx y Extr tienen una correlación negativa $(-0.308$ y -0.278 , respectivamente).

\section{DISCUSIÓN}

Dados los resultados obtenidos, se interpretan diferencias derivadas del análisis de la estadística descriptiva de las respuestas otorgadas por los participantes contra el modelo de regresión lineal. Es decir, lo que los alumnos perciben como importante para el rendimiento académico (el valor percibido de la tarea, la creencia de control y la motivación intrínseca) es diferente con respecto a las variables predictivas identificadas por el modelo (ansiedad al examen, aprendizaje por pares, motivación extrínseca, autoeficacia y regulación del esfuerzo). En otras palabras, lo que los alumnos creen no influye realmente en lo que obtendrán como calificación.
Es pertinente mencionar que el supuesto inicial de este estudio -esto es, que los alumnos con mayor motivación intrínseca y mejores habilidades metacognitivas serían quienes obtendrían un mejor rendimiento- no fue corroborado con los resultados obtenidos, ya que fueron las variables de aprendizaje por pares y la autoeficacia las que predicen en mayor medida el rendimiento académico (además de la ansiedad al examen y la motivación extrínseca, pero con una correlación negativa, y en menor medida la regulación del esfuerzo). Dicho de otra forma, en estudiantes novatos de programación influye en mayor grado la confianza en el propio éxito y el aprendizaje colaborativo que las habilidades metacognitivas.

Estos resultados son consistentes con estudios previos (Ramalingam, LaBelle, \& Wiedenbeck, 2004; Wiedenbeck, LaBelle, \& Kain, 2004) que señalan a la autoeficacia como un factor que afecta el rendimiento académico de programadores novatos, pero cabe mencionar que otros autores (Heggestad \& Kanfer, 2005) proponen que la autoeficacia es solo un efecto estadístico residual de la experiencia previa de la programación (figura 2).

Finalmente, la variable metacognitiva (Meta, autorregulación metacognitiva, tabla 2) no fue significativa ni en la percepción de utilidad de los 


\section{IIVESTIGAGIÓn Y CUERCIA DE LA UחIVERSIDAD AUTÓNOMA DE AGUASCALIERTES}

participantes, ni en las correlaciones, ni en el modelo de regresión generado. Esto puede interpretarse dada la evidencia empírica de otros estudios relacionados con el tema (Azevedo \& Aleven, 2013; Forbes-Riley \& Litman, 2010) como una falta de desarrollo de las habilidades metacognitivas en los estudiantes de este nivel inicial de estudios.

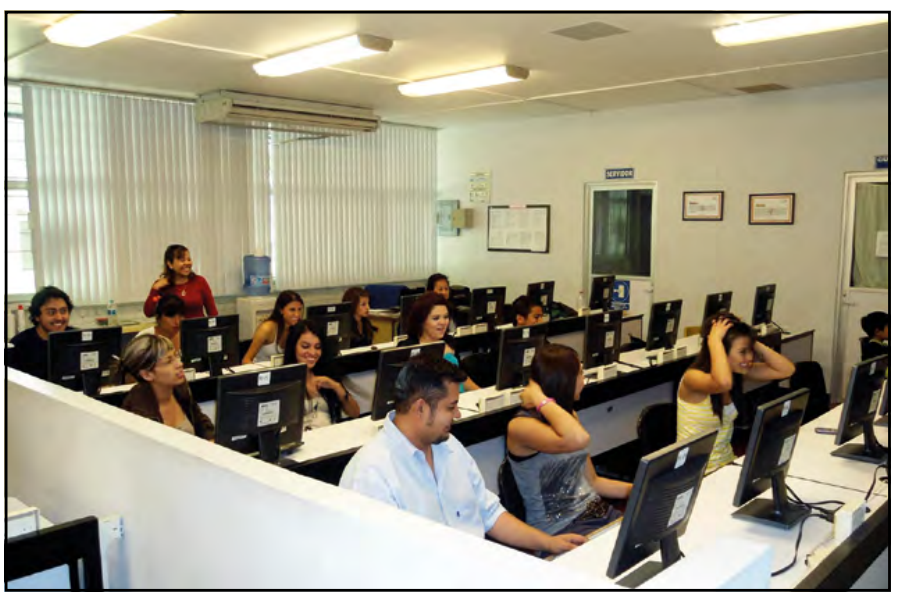

Figura 2. Aprendizaje de la programación.

Imagen tomada del portal de Facebook del Departamento de Sistemas de Información de la UAA (2014).

\section{CONCLUSIONES}

Trabajos futuros pueden ir orientados a identificar la relevancia del componente metacognitivo en estudiantes de ciencias computacionales después del primer año de estudios para observar si dichas habilidades metacognitivas están más desarrolladas, ya que el componente motivacional (en la forma de autoeficacia, ansiedad al examen y motivación extrínseca) si está presente como predictor desde el inicio de sus estudios universitarios.

Intervenciones didácticas para promover la autoeficacia se pueden extraer de la propia teoría (Bandura, 1982). Un catálogo de estrategias específicas asociadas con ella puede ser material de subsecuentes artículos, pero en general se recomienda incrementar la autoeficacia buscando que la teoría y la práctica en el salón de clases no sean demasiado avanzadas para la percepción de capacidad del estudiante, el cual debe ser retado, quien es probable que se rinda si se siente abrumado. La autoeficacia del estudiante se puede aumentar promoviendo éxitos en el rendimiento al solucionar tareas frecuentes con dificultad progresiva y marginalmente mayor, hasta que éste tenga una percepción sólida de logros.

\section{REFERENCIAS}

- Alexander, P. A. (2008). Why this and why now? Introduction to the special issue on metacognition, self-regulation, and self-regulated learning. Educational Psychology Review, 20, 369-372.

- Arévalo Mercado, A. C., Muñoz Andrade, E. L., \& Gómez Reynoso, J. M. (2011). A software tool to visualize verbal protocols to enhance strategic and metacognitive abilities in basic programming. International Journal of Interactive Mobile Technologies (iJIM), 5(3), 12-19.

- Azevedo, R., \& Aleven, V. (Eds.). (2013). Overview of current interdisciplinary research. En Metacognition and learning technologies (pp. 1-16). New York: Springer International Handbooks of Education.

- Baldwin, L. P., \& Macredie, R. D. (1999). Beginners and programming: Insights from second language learning and teaching. Education and Information Technologies, 4 (2), 167-179.

- Bandura, A. (1982). Self-efficacy mechanism in human agency. American Psychologist, 37(2), 122-147.

- Barak, M., Kastelan, I., \& Azia, Z. (2016). Exploring aspects of self-regulated learning among engineering students learning digital system design in the FPGA environment-methodolo- gy and findings. En R. Szewczyk, I. Kaštelan, M. Temerinac, M. Barak, \& V. Sruk (Eds.), Embedded Engineering Education (pp. 139-160). Springer International Publishing. doi: 10.1007/978-3319-27540-6_10

- Blackwell, A. F. (2002). First steps in programming: A Rationale for attention investment models. Proceedings IEEE 2002 Symposia on Human Centric Computing Languages and Environments, HCC 2002, 2-10.

- Bornat, R., Dehnadi, S., \& Simon. (2008). Mental models, consistency and programming aptitude. Proc. Tenth Australasian Computing Education Conference, ACE 2008, 53-62.

- Byrne, P., \& Lyons, G. (2001). The effect of student attributes on success in programming. ACM SIGCSE Bulletin, 33(3), 49-52.

- Cooper, S., Dann, W., \& Pausch, R. (2000). Alice: A 3D tool for introductory programming concepts. Journal of Computing Sciences in Colleges, 15(5), 107-116.

- Dehnadi, S., \& Bornat, R. (2006). The camel has two humps (working title) (pp. 1-21). London: Middlesex University. Recuperado de http://www.eis.mdx.ac.uk/research/PhDArea/ saeed/paperl.pdf 
- Dent, A. L., \& Koenka, A. C. (2016). The relation between self-regulated learning and academic achievement across childhood and adolescence: A meta-analysis. Educational Psychology Review, 28(3), 425-474. doi: 10.1007/s10648-0159320-8

- Departamento de Sistemas de Información UAA. (18 de febrero de 2014). [Foto del perfil en Facebook]. Recuperada de https://scontent.fntr4-1.fna.fbcdn.net/v/†31.08/1796885_1405880379669853_144507141_o.jpg?_nc_cat=0\& $\mathrm{oh}=1 \mathrm{f} 68 \mathrm{~b} 05 \mathrm{a} 6 \mathrm{~d} 1 \mathrm{~b} 8 \mathrm{dd} 2 \mathrm{afc} 2 \mathrm{c} 9 \mathrm{fd} 67 \mathrm{c} 6 \mathrm{f} 6 \mathrm{~d} 1 \mathrm{1} \& \mathrm{oe}=5 \mathrm{~B} 9347 \mathrm{F0}$

- Dewey, J. (1913). Interest and effort in education. Boston: Riverside.

- (1933). How we think. A restatement of the relation of reflective thinking to the educative process. Boston, MA: D. C. Heath. Brilliant.

- Flavell, J. H. (1979). Metacognition and cognitive monitoring: A new area of cognitive-developmental inquiry. American Psychologist, 34(10), 906-911.

- Forbes-Riley, K., \& Litman, D. (2010). Metacognition and learning in spoken dialogue computer tutoring. En Intelligent tutoring systems (pp. 379-388). Berlin-Heidelberg-New York: Springer-Verlag.

- Heggestad, E. D., \& Kanfer, R. (2005). The predictive validity of self-efficacy in training performance: Little More than past performance. Journal of Experimental Psychology: Applied, 11 (2), 84-97. doi: 10.1037/1076-898X.11.2.84

- Jenkins, T. (2002). On the difficulty of learning to program. Loughborough, Leicestershire, UK: Loughborough University.

- Kuhl, J. (2000). A functional-design approach to motivation and self-regulation: The dynamics of personality systems and interactions. En M. Boekaerts, P. R. Pintrich, \& M. Zeidner (Eds.), Handbook of self-regulation (pp. 111-169). San Diego, CA, US: Academic Press.

- Mahmoud, Q. H., Dobosiewicz, W., \& Swayne, D. (2004). Making computer programming fun and accessible. Computer, 37(2), 106-108. doi: 10.1109/MC.2004.1266305

- Maries, A., \& Kumar, A. (2007). Concept maps in intelligent tutors for programming. Journal of Computing Sciences in Colleges, $22(3), 54$.

- Mayer, R. E. (1988). The psychology of how novices learn computer programming. En E. Soloway, \& J. C. Spohrer (Eds.), Studying the novice programmer (pp. 129-159). Hillsdale, NJ: Lawrence Erlbaum Associates.
nal aspects of problem solving. Instructional Science, 26(1-2), 49-63. (2010). Applying the science of learning. Pearson.

- Milne, I., \& Rowe, G. (2002). Difficulties in learning and teaching programming-views of students and tutors. Education and Information Technologies, 7(1), 55-66.

- Mow, I. T. C. (2008). Issues and difficulties in teaching novice computer programming. Innovative Techniques in Instruction Technology, E-learning, E-assessment, and Education, 199204.

- Pintrich, P. R., Smith, D. A. F., Garcia, T., \& McKeachie, W. J. (1991). A manual for the use of the motivated strategies for learning questionnaire (MSLQ) [Documento en pdf]. Ann Arbor, Ml: The University of Michigan. Recuperado de https:// files.eric.ed.gov/fulltext/ED338122.pdf

- Ramalingam, V. L., La Belle, D., \& Wiedenbeck, S. (2004). Selfefficacy and mental models in learning to program. ITICSE '04 Proceedings of the $9^{\text {th }}$ Annual SIGCSE Conference on Innovation and Technology in Computer Science Education, UK, 2004, 171-175. doi: 10.1145/1007996.1008042

- Ryan, R. M., \& Deci, E. L. (2000). Intrinsic and extrinsic motivations: Classic definitions and new directions. Contemporary Educational Psychology, 25(1), 54-67.

- Schiefele, U. (1991). Interest, learning, and motivation. Educational Psychologist, 26(3-4), 299-323.

- Skubch, H., \& Thielscher, M. (2005). Strategy Learning for Reasoning Agents. En J. Gama, R. Camacho, P. Brazdil, A. Jorge, \& L. Torgo (Eds.), Machine Learning: ECML 2005 (pp. 733-740). Berlin-Heidelberg: Springer.

- Volet, S. E., \& Lund, C. P. (1994). Metacognitive instruction in introductory computer programming: A better explanatory construct for performance than traditional factors. Journal of Educational Computing Research, 10(4), 297-328.

- Wiedenbeck, S., LaBelle, D., \& Kain, V. N. R. (2004). Factors affecting course outcomes in introductory programming. En E. Dunican, \& T. R. G. Green (Eds.), Proceedings of the Psychology of Programming Interest Group 16 (pp. 97-110). Ireland: PPIG.

- Wong, L.-H., Chai, C.-S., Chen, W., \& Chin, C.-K. (2013). Measuring singaporean students' motivation and strategies of bilingual learning. The Asia-Pacific Education Researcher, 22 (3), 263-272. doi: 10.1007/s40299-012-0032-2 